\title{
Macrolide-resistant Mycoplasma genitalium infections in Cuban patients: an underestimated health problem
}

\author{
Brian Arturo Mondeja', Javier Couri ${ }^{1}, 2$, Nadia Maria Rodríguez'1 , Orestes Blanco ${ }^{1}$, Carmen Fernández ${ }^{1}$
} and Jørgen Skov Jensen ${ }^{3^{*}}$ (D)

\begin{abstract}
Background: The increasing prevalence of macrolide resistant Mycoplasma genitalium is a major concern worldwide. In Cuba, several cases of clinical treatment failure with $1 \mathrm{~g}$ single dose and extended azithromycin regimen have been detected and the aim of the present investigation was to retrospectively determine the prevalence of macrolide-resistance mediating mutations (MRMM) in M. genitalium-positive samples conserved at the Cuban National Reference Laboratory of Mycoplasma Research between 2009 and 2016.

Methods: A total of 280 positive DNA extracts were analysed by a 5' nuclease assay for detection of $M$. genitalium MRMM. Ten urogenital specimens from patients with azithromycin treatment failure and MRMM were inoculated in Vero cell to obtain the isolates for subsequent determination of antimicrobial susceptibility.

Results: The overall prevalence of MRMM was 32\%. No MRMM was detected in samples collected between 2009 and 2013 but since 2014 a dramatic increase to 90\% (95\% Cl, 76-96\%) in 2016 was seen. Three new M. genitalium isolates were isolated in Vero cell cultures and confirmed phenotypic resistance to macrolides in a cell-culture assisted susceptibility test. Preliminary observations suggest that combination therapy with levofloxacin and doxycycline may represent an affordable option for treatment of macrolide resistant $M$. genitalium infections.

Conclusions: This investigation showed the rapid emergence and high prevalence of MRMM in M. genitalium-infected patients in Cuba and confirmed the phenotypic resistance in isolates carrying MRMM. We suggest that Cuban guidelines for sexually transmitted infections are modified to include testing for $M$. genitalium and detection of MRMM in patients with failure of syndromic treatment, to ensure that in these cases, the treatment will be guided by etiologic diagnosis.
\end{abstract}

Keywords: Mycoplasma genitalium, Antimicrobial susceptibility, Macrolide resistance, Sexually transmitted infections

\section{Background}

Mycoplasma genitalium is an emerging sexually transmitted pathogen and the increase of macrolide resistance is considered a health problem globally [1]. In this bacterium, macrolide resistance is mediated mainly by point mutations in the A2058 and A2059 (Escherichia coli numbering) positions in region $\mathrm{V}$ of the $23 \mathrm{~S}$ ribosomal RNA. These mutations are associated with azithromycin treatment failure and high minimal inhibitory concentration (MIC) for

\footnotetext{
* Correspondence: jsj@ssi.dk

${ }^{3}$ Statens Serum Institut, Research Unit for Reproductive Tract Microbiology, Artillerivej 5, DK-2300 Copenhagen S, Denmark

Full list of author information is available at the end of the article
}

macrolides as documented in several M. genitalium strains $[2,3]$.

The prevalence of strains with macrolide resistance mediating mutations (MRMM) is highly variable and unknown in many regions. In Europe, Australia and Asia, it is generally above $30 \%$ and in extreme cases, as in Greenland, $100 \%$ of M. genitalium strains carry MRMM [4-8]. In Cuba, macrolides are used as the first line treatment for urogenital syndromes caused by sexually transmitted infections (STI), including M. genitalium and chlamydia infections. Since 2007, M. genitalium diagnosis has been performed at the Pedro Kourí Tropical Medicine Institute (IPK), and several cases of treatment failure with $1 \mathrm{~g}$ single dose and extended azithromycin regimens have

(c) The Author(s). 2018 Open Access This article is distributed under the terms of the Creative Commons Attribution 4.0 International License (http://creativecommons.org/licenses/by/4.0/), which permits unrestricted use, distribution, and reproduction in any medium, provided you give appropriate credit to the original author(s) and the source, provide a link to the Creative Commons license, and indicate if changes were made. The Creative Commons Public Domain Dedication waiver (http://creativecommons.org/publicdomain/zero/1.0/) applies to the data made available in this article, unless otherwise stated. 
been detected in the IPK - STI clinic within recent years. In 2015, a new macrolide resistant $M$. genitalium strain (B19, A2059G mutation) was isolated from one of these patients using Vero cell co-culture [9], and antimicrobial susceptibility patterns were determined using a cellassisted procedure [10]. However, no data about the prevalence of MRMM carrying $M$. genitalium strains in Cuba are available, but clinical experience of macrolide treatment failures and the isolation of at least one MRMM strain suggest the possible circulation of MRMM carrying M. genitalium strains in Cuban patients after 2015, which has direct implications for the effectiveness of syndromic management of STI.

The aim of the present investigation was to retrospectively determine MRMM prevalence in M. genitalium-positive samples conserved at the Cuban National Reference Laboratory of Mycoplasma Research on IPK between 2009 and 2016.

\section{Materials and methods Study design}

A retrospective study was conducted at the Cuban National Reference Laboratory of Mycoplasma Research - IPK, for the direct detection of MRMM in M. genitalium positive clinical samples archived since 2009. This study was approved by The Pedro Kourí Tropical Medicine Institute Ethical Board (approval CEI-IPK 57-16) and written informed consent to participate and publish was obtained from all patients.

\section{M. genitalium positives specimens}

For the study, a total of $280 \mathrm{M}$. genitalium positive DNA extracts from Cuban patients with urogenital syndromes, spontaneous abortion and infertility were analysed. These were representing all samples submitted to IPK for M. genitalium diagnosis since 2009 and up to December 2016. DNA was extracted by the Chelex 100 method and conserved at $-80{ }^{\circ} \mathrm{C}$ [11]. Until December of 2014, M. genitalium diagnosis was performed by a $16 \mathrm{~S}$ rRNA singleplexPCR with internal control [12] and confirmation by a qPCR based in the amplification of $m g p B$ gene [11]). Since January of $2015, M$. genitalium diagnosis was performed by the same $m g p B$ gene qPCR mentioned above and the confirmation was with a $m g p A$ gene - qPCR [13]. For each patient, only the first positive specimen was included in the study.

\section{Detection of MRMM}

DNA specimens were analyzed by a modification of the $5^{\prime}$ nuclease genotyping assay for M. genitalium MRMM testing, described by Kristiansen et al. [14]. In brief, a qPCR mix was prepared in a final volume of $25 \mu \mathrm{L}$ containing $500 \mathrm{nM}$ of the 23S rRNA gene primers described by Jensen et al. [15], $200 \mathrm{nM}$ of wild-type probe: Cy5-GGA CGG AAA GAC CCC GTG AAG CTT T-BHQ2, $100 \mathrm{nM}$ of each MRMM probes MRMM-A2058G: FAM-GAC GGG AAG ACC CCG TGA AGC TTT-BHQ1 and MRMMA2059G: FAM- GAC GGA GAG ACC CCG TGA AGC TTT-BHQ1 [14], and 1.5 U of TopTaq-polymerase (Qiagen,Hilden, Germany). As positive controls for MRMM, DNA from $M$. genitalium strains M6271 (A2058G), M6489 (A2059G) and M6302 (A2058C) were used, and as wild-type M. genitalium strains the Danish strains M2300, M2321 and M2341 were used [3].

Samples were amplified and analysed in a Rotor-Gene $Q$ 5-plex instrument (Qiagen) using the same amplification program as described by Kristiansen et al. [14]. Results were analysed using the Rotor-Gene Q Software Version 2.1.0.9 (Qiagen) by the Scatter Analysis module with the reading combination of the green and red fluorescence channel.

Isolation and determination of antimicrobial susceptibility of MRMM strains from clinical specimens

Ten urogenital specimens from patients with azithromycin treatment failure and with an $M$. genitalium load > 10,000 genome equivalents (geq) per $\mathrm{mL}$, were inoculated in Vero cell as previously described [16] in an attempt to obtain new isolates. The specimens comprised seven male urethral swabs (three male homosexual couples and one heterosexual man) and three endocervical swabs (one of them from the female partner of the male patient providing the sample yielding the B19 strain previously described [10]). In order to document lack of cross-contamination between cell cultures, an $m g p B$ based DNA typing method [17] was applied to primary samples and isolated strains.

Antimicrobial susceptibility testing was performed by a cell-assisted procedure as previously described, but with an incubation time of 28 days [10]. MICs to azithromycin, erythromycin, doxycycline, tetracycline, ciprofloxacin, ofloxacin, moxifloxacin and levofloxacin was defined as the lowest concentration of antimicrobial capable of inhibiting 99\% of the M. genitalium growth in the test well compared with the $M$. genitalium growth in the control wells [18]. MRMM identification of the isolates was done by the nuclease genotyping assay described above, using genomic DNA of the isolate and of the corresponding clinical specimen.

\section{Treatment of patients with macrolide resistant $M$. genitalium infection}

Between July 2015 and December 2017, all 46 M. genitalium positive patients with treatment failure after azithromycin (500 mg day 1 followed by $250 \mathrm{mg}$ days 2-5) and attending the STD clinic of the IPK Hospital have been treated with doxycycline $100 \mathrm{mg} 2$ times daily plus levofloxacin $500 \mathrm{mg} 2$ times daily for 14 days. This regimen has been used due to the limited availability of moxifloxacin in Cuba. Microbiological cure was evaluated 30 days 
after treatment using the same PCR methodology as used for the primary diagnosis.

\section{Statistical analysis}

The statistical comparisons between the frequency of resistant M. genitalium in each year of the study and for the proportion of MRMM between men and women was compared with Fisher's exact test with $\mathrm{p}$ for trend (two-sided $p$ values). Differences with $p<0.05$ were considered statistically significant (StatsDirect version 3.1.14 (StatsDirect Ltd., Cheshire, UK)).

\section{Results}

\section{Distribution of $M$. genitalium positive patients}

Of the $280 \mathrm{M}$. genitalium positive patients, 100 (35.7\%) were men and 180 (64.3\%) were women (Fig. 1).

\section{Prevalence of MRMM in the clinical specimens}

Of the 280 samples analysed, 202 (72\%) were amplifiable by the MRMM qPCR. Of these 202, 138 (68\%; 95\% CI 57-81\%) were identified as wild-type genotype (macrolide susceptible) and 64 (32\%; 95\% CI 24-40\%) were identified as carrier of mutant genotypes. MRMM was not detected in any of the 65 successfully amplified samples collected between 2009 and 2013 0\% (95\% CI: 0-6\%). In contrast, MRMM was detected in 64 of 137 clinical samples $47 \%$ (95\% CI: 39-55\%) collected since 2014 with a dramatic increase to $90 \%$ (95\% CI: 76-96\%) in $2016(p<0.0001$, $p<0.0001$ for trend) (Fig. 2).

No differences between the MRMM prevalence in men and women were detected $(p=0.88)$.

\section{Genotype distribution among MRMM positive samples} Out of a total of 64 MRMM positive samples, 52 samples (81\%) were identified as A2058G/A2059G and 12

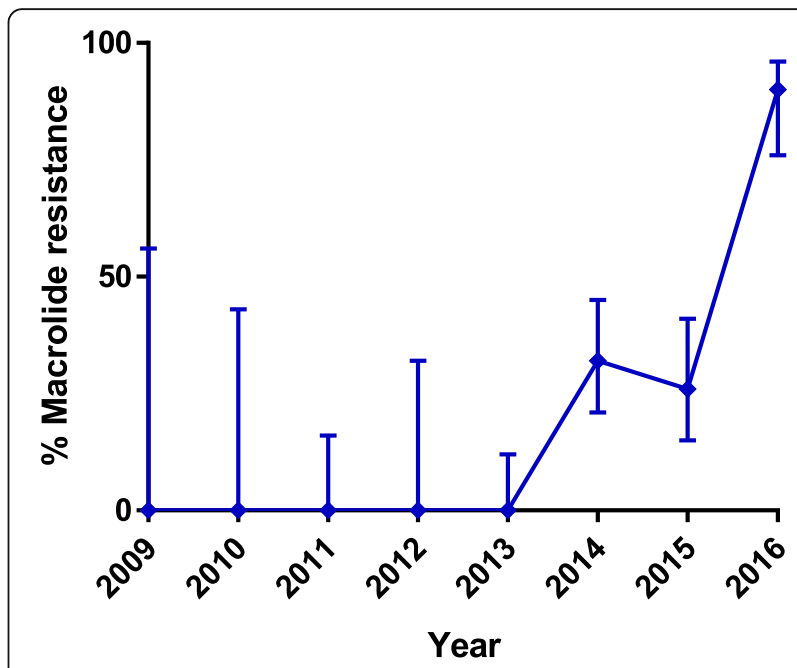

Fig. 2 Prevalence of MRMM genotype in percent of clinical specimens per year of study. Error-bars show 95\% confidence intervals

(19\%) as A2058C/T. The latter genotype was more commonly found in female than in male specimens.

\section{Antimicrobial susceptibility pattern of MRMM strains from clinical specimens}

Of the ten $M$. genitalium positive specimens, only three were capable of growing in the Vero cell culture and yielded new isolates. The B25 and B26 strains were isolated from a male couple and showed the same $m g p B$ type (not previously described and denominated Cuba3; Acc\# MH407699). The B30 strain was isolated from the female partner of the male patient yielding the B19 isolate previously reported [10]. The B30 strain showed an identical $m g p B$ type to that of the B19 strain (genotype 4). For all three isolates, the same $m g p B$ type was detected from isolate and the corresponding clinical specimen.

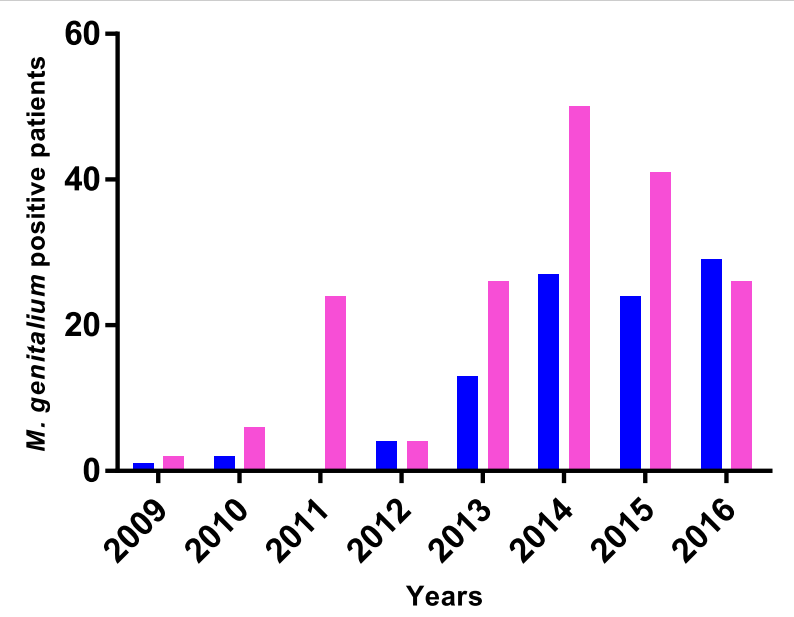

Fig. 1 Gender distribution and number of M. genitalium positive patients per year of study 
The three new clinical $M$. genitalium isolates were resistant to macrolides with a MIC value of $>8 \mathrm{mg} / \mathrm{L}$. MIC values for tetracyclines were $0.5-2 \mathrm{mg} / \mathrm{L}$ and for quinolones, a MIC of $0.125 \mathrm{mg} / \mathrm{L}$ was found for moxifloxacin (Table 1).

All the isolates and the corresponding clinical specimen were identified as MRMM A2058G/A2059G by the 5 ' nuclease assay and as A2059G by sequencing (Table 1) clearly confirming the good concordance between detection of MRMM by the molecular assay and the demonstration of a phenotypic resistance in vitro in the cell-assisted assay as well as in vivo documented by macrolide treatment failure.

\section{Treatment of patients with macrolide resistant $M$. genitalium infection}

All 46 cases with azithromycin treatment failure were found to carry $M$. genitalium with MRMM. Dual therapy with doxycycline and levofloxacin for 14 days eradicated M. genitalium from all patients as documented by PCR 30 days after treatment.

\section{Discussion}

The emergence of MRMM in M. genitalium in several countries is a worrying reality. For several years, this sexually transmitted pathogen has been underestimated as responsible for STI syndromes in men and women. In the Unites States Center for Diseases Control STI treatment guidelines the infection got attention with a separate chapter for the first time in 2015 [19]. In 2016 the European guideline on Mycoplasma genitalium infections was published as the first international guideline addressing diagnosis and treatment [20]. This was the first step to harmonize the international recognition and treatment of $M$. genitalium as an STI.

Since 2004, M. genitalium infections have been studied at IPK, and diagnosis was established in 2009 as part of an aetiological surveillance of STIs in Cuba. For many years, empiric treatment with macrolides and tetracyclines was used for the control of mycoplasma and chlamydia infections. In 2001, Cuba implemented syndromic management of STI, and the $1 \mathrm{~g}$ single dose azithromycin treatment became the recommended treatment for non-gonococcal urogenital infections in men and women. At this time, few studies were available that evaluated the treatment efficacy of macrolides and the emergence of resistance was an unknown problem. The latter, has become recognised as a worldwide problem after 2008 [2].

In the present investigation, we have determined the presence of MRMM in M. genitalium in Cuban clinical specimens archived since 2009, nine years after the initiation of azithromycin syndromic treatment of urogenital syndromes, and until 2016. From our data, it appears that $M$. genitalium with MRMM was very rare in Cuba before 2014. However, the number of analysed M. genitalium positive samples between 2009 and 2013 was relatively low, so it is difficult to make definite conclusions. Nevertheless, the explosion in the prevalence of M. genitalium with MRMM reaching $90 \%$ in 2016 is extremely worrying and threatening the current recommended syndromic management. In Cuba, more than 4000 cases of urogenital syndromes (lower abdominal pain, vaginal discharge and urethral syndrome) are reported to the Ministry of Public Health every year. However, it is not common that $M$. genitalium tests are ordered, even when macrolide treatment has failed. This finding is very similar in other countries and is partly due to lack of awareness among clinicians, but also due to lack of testing availability in many settings.

The genotyping assay used for MRMM detection was able to characterise more than $70 \%$ of the M. genitalium positive specimens, compared with $94.5 \%$ reported in the original paper by Kristiansen et al. [14]. However, when we analysed samples from 2016, 15 of the 17 non-amplified specimens were successfully re-amplified by the $m g p B$ qPCR. These samples showed an M. genitalium load $<1000$ geq $/ \mathrm{mL}$ which may explain the failure of amplification in the genotyping assay. Other factors such as destruction of DNA, even when conserved at $-80{ }^{\circ} \mathrm{C}$, could be playing a role in the success of the genotyping assay, as has been shown for another M. genitalium molecular test [21]. Unfortunately, it was not possible to provide information about the exact positions of the MRMM as the method applied did not provide this level of discrimination. Most of the samples were used up for culture attempts and additional PCRs for MRMM was not possible. In Cuba, Sanger sequencing is difficult and expensive and consequently, a qPCR for the detection of MRMM directly from the clinical samples

Table 1 Minimal Inhibitory Concentrations (MICs) for the three MRMM carrying Cuban M. genitalium isolates as determined by a modified cell-culture-based method

\begin{tabular}{llllllllll}
\hline M. genitalium isolates & \multicolumn{1}{l}{ MIC VALUES (mg/L) } \\
\hline B25 & MRMM & Azithromycin & Erythromycin & Ciprofloxacin & Ofloxacin & Levofloxacin & Moxifloxacin & Tetracycline & Doxycycline \\
B26 & A2059G & $>8$ & $>8$ & 4 & 1 & 2 & 0.125 & 1 & 0.5 \\
B30 & A2059G & $>8$ & $>8$ & 4 & 1 & 0.5 & 0.125 & 1 & 0.5 \\
\hline
\end{tabular}


was introduced. Furthermore, as the type of mutation is not relevant for the clinical management, and as an increasing amount of surveillance data is produced by PCR based testing, even in more resourced settings, we do not consider this to be a major limitation of the study.

The three new Cuban $M$. genitalium strains isolated from samples with MRMM have an antimicrobial resistance profile with susceptibility to tetracyclines and moxifloxacin according to CLSI guidelines for $M$. pneumoniae [22]. The patient from whom the B25 strain was isolated is a Cuban man living in Switzerland, where he was infected, diagnosed and treated for M. genitalium. His Cuban male partner never lived outside of Cuba and was infected after unprotected sex during a visit of patient B25. Thus, introduction of the MRMM M. genitalium strain from Europe is the most plausible explanation, and confirmation of the same $m g p B$ type (Cuba3) in clinical samples and MRMM strains, support this. Fortunately, both the B25 and B26 strains were susceptible to doxycycline and the patients were cured after combined treatment with doxycycline and levofloxacin for 14 days. The B30 female patient was a woman having had sex with foreign men and she was the sexual contact reported by the B19 male patient described in our previous study [10] at the time of $M$. genitalium diagnosis. The same $m g p B$ type (type 4 ) was found in the clinical specimens and isolated strains B19 and B30, reaffirming the sexual transmission between the partners. However, it is impossible to determine if the MRMM strain was imported or not. Both patients were cured with levofloxacin plus doxycycline.

All three new isolates showed phenotypic resistance to macrolides. In these cases, a total concordance between the 5'nuclease genotyping assay, the macrolide resistance pattern determined by the Vero cell culture assay, and clinical and microbiological failure of azithromycin treatment was documented. This fact reaffirm the utility of the genotyping assay to predict azithromycin treatment failure in patients infected with $M$. genitalium when MRMM are detected. Unfortunately, no isolates with MRMM were obtained from the seven other clinical specimens inoculated in Vero cells.

In this investigation, the overall macrolide resistance rate was $32 \%$ but with all of the resistant strains detected within the last three years of the study. The A2058G/A2059G was the most frequently detected MRMM genotype detected in M. genitalium positives specimens as in most other studies. Comparable rates of macrolide resistance have been reported recently from Denmark [4], United Kingdom [6], and Australia [8], where the prevalence of macrolide resistance-associated mutations was 40, 41 and 43\%, respectively. These studies also found similar distributions of the most common A2058G and A2059G mutations but did not see the same dramatic increase in the prevalence of MRMM strains. The A2058C/T was detected in $19 \%$ of Cuban MRMM specimens. Unfortunately, neither of the isolated strains had this mutation pattern but no influence on the MICs values for macrolides have been recorded in other investigations for the different mutation-types [2, 3].

Based on the findings reported here, a new molecular diagnostic strategy for $M$. genitalium at IPK has been established since January 2017. In this strategy, we use the $m g p \mathrm{~B}$ qPCR as screening test and positive specimens are immediately confirmed by the 5 'nuclease genotyping assay and by the $m g p A$ qPCR for $m g p B$ positive samples with less than $1000 \mathrm{geq} / \mathrm{mL}$. This approach is in accordance with the European guideline on M. genitalium infections [20] where determination of MRMM is recommended for all $M$. genitalium positive samples.

The prevalence of MRMM carrying M. genitalium strains has been high after 2014 in Cuba. Since 2015, all M. genitalium positive patients with MRMM seen at the STD clinic at the IPK Hospital have demonstrated treatment failure after azithromycin. In these 46 cases, MRMM M. genitalium was successfully eradicated with a 14-days second-line combined therapy of levofloxacin plus doxycycline (Orestes Blanco, unpublished). This regimen has been used due to the limited availability of moxifloxacin in Cuba and may be applicable elsewhere. The choice of this combination regimen was based on the synergistic in vitro activity of doxycycline and moxifloxacin in fluoroquinolone susceptible M. genitalium strains [23]. Experimental work documenting the synergy between levofloxacin and doxycycline is currently underway. The high efficacy was somewhat unexpected as doxycycline monotherapy has a microbiological cure rate of approximately $30 \%$ and that of levofloxacin monotherapy is rarely exceeding 50\% [24].

No data about MRMM prevalence in other Latin-American countries are available, but it will probably be found at a similar rate in countries with similar macrolide treatment strategy for STIs as Cuba.

\section{Conclusions}

The results obtained in this investigation showed for the first time the rapid emergence and high prevalence of MRMM carrying M. genitalium strains in Cuban patients. No data about macrolide failure in syndromic STI management outside IPK are available, but macrolide resistance in $M$. genitalium-infected Cuban patients could be an underestimated health problem. We suggest that Cuban guidelines for STI treatment are modified to include testing for $M$. genitalium and detection of macrolide resistance-mediating mutations at least in patients failing syndromic treatment to ensure that in these cases the treatment will be guided by the aetiological diagnosis. 


\section{Abbreviations}

IPK: Pedro Kourí Tropical Medicine Institute; MRMM: Macrolide resistance mediating mutations; qPCR: Quantitative Polymerase Chain Reaction; STI: Sexually transmitted infections

\section{Acknowledgements}

Lic. Ruxana Sardiñas provided excellent technical assistance with M. genitalium PCR diagnosis.

\section{Consent to publication}

Written informed consent to participate and publish was obtained from all patients.

\section{Funding}

No specific funding was received for this study. The work was supported in part by the Pedro Kourí Tropical Medicine Institute, Cuba, with the collaboration and economical support of Statens Serum Institut, Denmark. No funding bodies had any role in study design, data collection and analysis, decision to publish, or preparation of the manuscript.

\section{Availability of data and materials}

The datasets used and/or analysed during the current study are available from the corresponding author on reasonable request.

\section{Authors' contributions}

Conceptualization: BAM JSJ. Data curation: BAM JC OB JSJ. Formal analysis: BAM JSJ. Funding acquisition: BAM NMR JSJ. Investigation: BAM JC OB CF JSJ. Methodology: BAM NMR CF JSJ. Project administration: BAM NMR. Resources: JSJ OB NMR. Supervision: JSJ NMR CF. Validation: NMR. Visualization: BAM NMR JSJ. Writing - original draft: BAM JSJ. Writing review\& editing: BAM NMR JC OB CF JSJ.All authors read and approved the final manuscript.

\section{Ethics approval and consent to participate}

This study was approved by The Pedro Kourí Tropical Medicine Institute Ethical Board (approval CEI-IPK 57-16) and written informed consent to participate and publish was obtained from all patients.

\section{Competing interests}

BM, JC, NM, OB, and CF report no conflict of interest. JSJ has received speaker's fee from Hologic and serves a scientific advisory board of Roche Molecular Systems. SSI has received remuneration for contract work from SpeeDx, Hologic, NYTor, Diagenode, Nabriva, GSK, and Angelini.The authors declare that they have no competing interest.

\section{Publisher's Note}

Springer Nature remains neutral with regard to jurisdictional claims in published maps and institutional affiliations.

\section{Author details \\ ${ }^{1}$ Pedro Kourí Tropical Medicine Institute, La Habana, Cuba. ${ }^{2}$ Faculty of Biology, University of Havana, La Habana, Cuba. ${ }^{3}$ Statens Serum Institut, Research Unit for Reproductive Tract Microbiology, Artillerivej 5, DK-2300 Copenhagen S, Denmark.}

Received: 6 March 2018 Accepted: 19 November 2018 Published online: 29 November 2018

\section{References}

1. Unemo M, Jensen JS. Antimicrobial-resistant sexually transmitted infections: gonorrhoea and Mycoplasma genitalium. Nat Rev Urol. 2017;14:139-52.

2. Jensen JS, Bradshaw CS, Tabrizi SN, Fairley CK, Hamasuna R. Azithromycin treatment failure in Mycoplasma genitalium-positive patients with nongonococcal urethritis is associated with induced macrolide resistance. Clin Infect Dis. 2008:47:1546-53.

3. Jensen JS, Fernandes P, Unemo M. In vitro activity of the new fluoroketolide solithromycin (CEM-101) against macrolide-resistant and - susceptible Mycoplasma genitalium strains. Antimicrob Agents Chemother. 2014;58:3151-6.

4. Salado-Rasmussen K, Jensen JS. Mycoplasma genitalium testing pattern and macrolide resistance: a Danish nationwide retrospective survey. Clin Infect Dis. 2014;59:24-30.
5. Gesink DC, Mulvad G, Montgomery-Andersen R, Poppel U, MontgomeryAndersen S, Binzer A, et al. Mycoplasma genitalium presence resistance and epidemiology in Greenland. Int J Circumpolar Health. 2012;71:1-8.

6. Pond MJ, Nori AV, Witney AA, Lopeman RC, Butcher PD, Sadiq ST. High prevalence of antibiotic-resistant Mycoplasma genitalium in nongonococcal urethritis: the need for routine testing and the inadequacy of current treatment options. Clin Infect Dis. 2014;58:631-7.

7. Nijhuis RH, Severs TT, Van der Vegt DS, Van Zwet AA, Kusters JG. High levels of macrolide resistance-associated mutations in Mycoplasma genitalium warrant antibiotic susceptibility-guided treatment. J Antimicrob Chemother. 2015;70:2515-8

8. Tagg KA, Jeoffreys NJ, Couldwell DL, Donald JA, Gilbert GL. Fluoroquinolone and macrolide resistance-associated mutations in Mycoplasma genitalium. J Clin Microbiol. 2013;51:2245-9.

9. Jensen JS, Hansen HT, Lind K. Isolation of Mycoplasma genitalium strains from the male urethra. J Clin Microbiol. 1996;34:286-91.

10. Mondeja BA, Rodriguez NM, Barroto B, Blanco O, Jensen JS. Antimicrobial susceptibility patterns of recent Cuban Mycoplasma genitalium isolates determined by a modified cell-culture-based method. PLoS One. 2016;11:e0162924.

11. Jensen JS, Björnelius E, Dohn B, Lidbrink P. Use of TaqMan 5' nuclease real-time PCR for quantitative detection of Mycoplasma genitalium DNA in males with and without urethritis who were attendees at a sexually transmitted disease clinic. J Clin Microbiol. 2004;42:683-92.

12. Jensen JS, Borre MB, Dohn B. Detection of Mycoplasma genitalium by PCR amplification of the 16S rRNA gene. J Clin Microbiol. 2003;41:261-6.

13. McGowin CL, Annan RS, Quayle AJ, Greene SJ, Ma L, Mancuso MM, et al. Persistent Mycoplasma genitalium infection of human endocervical epithelial cells elicits chronic inflammatory cytokine secretion. Infect Immun. 2012;80:3842-9.

14. Kristiansen GQ, Lisby JG, Schonning K. A 5' nuclease genotyping assay for identification of macrolide-resistant Mycoplasma genitalium in clinical specimens. J Clin Microbiol. 2016;54:1593-7.

15. Jensen JS. Protocol for the detection of Mycoplasma genitalium by PCR from clinical specimens and subsequent detection of macrolide resistancemediating mutations in region $V$ of the 235 rRNA gene. In: CR MK, Henrich B, editors. Diagnosis of Sexually Transmitted Diseases; Methods and Protocols. 903rd ed. New York: Humana Press, Springer; 2012. p. 129-39.

16. Mondeja BA, Jensen JS, Rodriguez I, Morier LF, Kouri V, Rodriguez NM, et al. Isolation of mycoplasma genitalium from patients with urogenital infections: first report from the Latin-American region. New Microbes New Infect. 2013;1:22-6.

17. Hjorth SV, Björnelius E, Lidbrink P, Falk L, Dohn B, Berthelsen L, et al. Sequence-based typing of Mycoplasma genitalium reveals sexual transmission. J Clin Microbiol. 2006:44:2078-83.

18. Hamasuna R, Osada $Y$, Jensen JS. Antibiotic susceptibility testing of Mycoplasma genitalium by TaqMan 5' nuclease real-time PCR. Antimicrob Agents Chemother. 2005:49:4993-8.

19. Workowski KA, Bolan GA. Sexually transmitted diseases treatment guidelines, 2015. MMWR Recomm Rep. 2015:64:1-137.

20. Jensen JS, Cusini M, Gomberg M, Moi H. 2016 European guideline on Mycoplasma genitalium infections. J Eur Acad Dermatol Venereol. 2016;30:1650-6.

21. Carlsen $\mathrm{KH}$, Jensen JS. Mycoplasma genitalium PCR: does freezing of specimens affect sensitivity? J Clin Microbiol. 2010;48:3624-7.

22. CLSI (Eds):Methods for antimicrobial susceptibility testing for human mycoplasmas; Approved guideline. In CLSI document 2011, M43-A.

23. Bradshaw CS, Jensen JS, Waites KB. New horizons in Mycoplasma genitalium treatment. J Infect Dis. 2017;216:S412-9.

24. Jensen JS, Bradshaw C. Management of Mycoplasma genitalium infections - can we hit a moving target? BMC Infect Dis. 2015;15:343. 